\title{
New Design and Fabrication Methods for Freeform Stone Vaults Based on Ruled Surfaces
}

Matthias Rippmann and Philippe Block

\section{Introduction}

Thin concrete and steel grid shells show elegantly how shell design is used for contemporary freeform architecture. Their natural beauty is coupled to an inherent efficiency due to minimal bending, result from their good structural form. Thanks to digital form finding tools, streamlined planning processes and automated fabrication, the technical and economic difficulties to design and build those structures, especially grid shells, decreased significantly [1].

In contrast, the use of stone as a structural material for elaborate and exciting freeform architecture did not develop in a similar manner. However, even today, Gothic cathedrals show the natural aesthetics of stone structures in an impressive way, combining structural and ornamental building parts to realize complex building forms [2]. The industrialized methods of processing stone, needed for freeform vaults, are less established and less flexible than comparable procedures for steel and concrete, for which a continuous and great improvement took place over the last century [3]. The new approaches shown in this paper aims to pave the way for the use of stone for freeform vault structures in the near future.

In this context, ruled surfaces are of particular interest due to the geometric configuration of discrete stone blocks (voussoirs) of freeform vaults, which originates both from the statics of vaults (Section 2.1), and from stone cutting based on wire-cutting technology (Section 2.2).

The use of ruled-surface design has been of special interest for freeform architecture, which comprises parts or patches of ruled surfaces, for example of hyperbolic paraboloids (hypar), rotational hyperboloids or helicoids [4]. Firstly, ruled surface based design is used to explicitly describe those forms, which dates back to the work of Antoni Gaudí, who was a pioneer in using ruled surfaces to define the complex geometry of his designs (fig. 1.1 a) [5].

Matthias Rippmann · Philippe Block

Institute of Technology in Architecture, ETH Zurich, Switzerland 
A second, well known use of ruled surfaces in the building process is for rationalizing the construction work for a building. Felix Candela for example took advantage of the fact that through every point on a ruled surface runs at least one straight line that lies on this surface. Therefore, he constrained his thin, concrete shells to combinations of parts of hypars which then could be built using formwork out of linear elements (fig. $1.1 \mathrm{~b}$, right). Moreover, doubly ruled surfaces can be used to apply planar quadrilateral panels on freeform architecture (fig. $1.1 \mathrm{~b}$, left) [4].

Thirdly, fabrication techniques based on ruled surfaces, such as diamond-wire for stone or hot-wire cutting for foam, typically process these material faster, with less kerf and material waste, than traditional subtractive manufacturing methods such as milling and solid blade machining [6]. For example, for the glass fiber reinforced concrete panels of the facade of the Cagliari Contemporary Arts Center by Zaha Hadid Architects hot-wire cutting was used to produce the EPS foam molds for the freeform panels (fig. $1.1 \mathrm{c}$ ) [7].

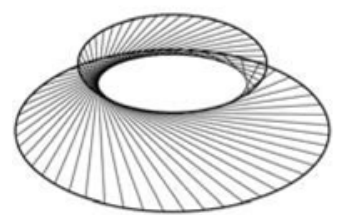

a
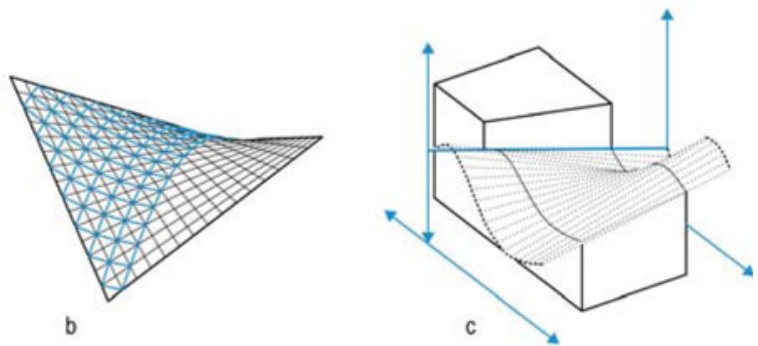

Fig. 1.1 (a) The definition of a hyperboloid by a moving line along two circles (b) A hyperbolic paraboloid consists of two families of intersecting lines and can be populated with planar quad meshes (c) A tensioned wire cuts through material, tracing a ruled surface

For the design and realization of freeform stone vaults we considered those strategies to consequently identify efficient ways of feasible construction. A key aspect was to define and develop a suitable and coordinated design and fabrication setup for the production of hundreds of individual voussoirs, which need to be processed for a single vault design. Due to the three-dimensional shape of the voussoirs and the geometrically complex fabrication constraints, the challenge is to coordinate the design of the individual voussoirs in combination with the technical machine setup. The challenge is to find the right balance of the integrated design and fabrication method to achieve a feasible configuration to produce freeform stone vaults efficiently. This potential of a well-coordinated digital design and fabrication setup for cutting stone to realize freeform stone vaults has not been tapped yet. Additionally, the software to design, simulate and process the part geometry is typically used one after the other, missing an integrated concept to cope with the above mentioned challenges.

In this paper, we show that new approaches of digital design and fabrication methods unveil great potential for building processes in the field of freeform stone vaults. Considering the materialization process of freeform vaults the research leads to a novel, streamlined approach, demonstrating the smooth integration of 
structural needs, fabrication constraints and economic feasibility into the design and production process.

\section{Geometrical Considerations}

In this section, the geometrical and technical information about discrete freeform vaults and state-of-the-art wire-cutting machine setups will be described. Also, the relevance of ruled surfaces for freeform stone vault design and fabrication will be described.

\subsection{Freeform Stone Vaults}

The design and realization of freeform vaults comprises four parts: form finding [8], tessellation [9], voussoir generation, also known as stereotomy [10], and vault assembly.

This Subsection focuses on the generation of the voussoirs, which is based on the tessellation and the surface geometry of the funicular form. Ideally, to prevent sliding failure and guarantee structural stability, the main load bearing faces of neighboring voussoirs need to be perpendicular to the force flow. Therefore, the interface surfaces should be normal to the thrust surface at any point. This can be best described by examining a single voussoir based on a hexagonal tessellation pattern applied on an arbitrary thrust (i.e compression-only) surface as shown in fig. 2.1.
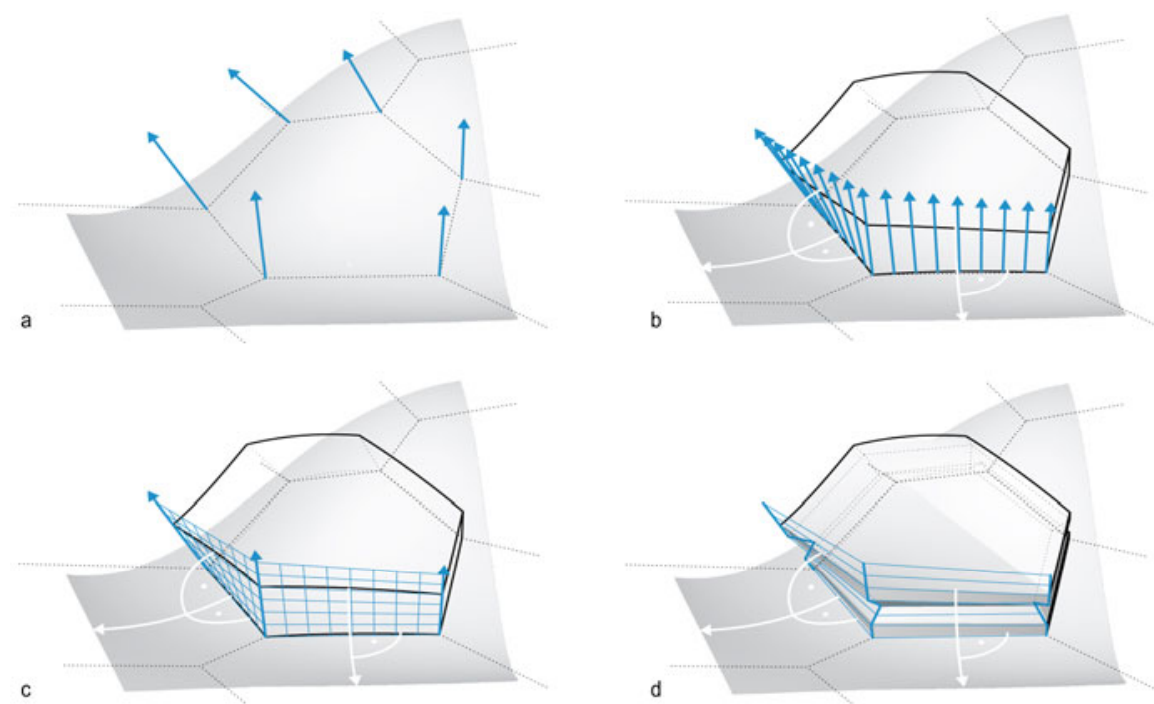

Fig. 2.1 (a) Surface normals define the vertical edges of voussoirs (b) A family of surface normals defines the load bearing surface (c) Lofting between two edge lines results in a doubly ruled surface (d) The horizontal orientation of the generators increases the geometric flexibility 
The tessellation pattern is represented by a set of points on the surface and a network of connecting lines. Considering the perpendicularity to the thrust surface, the orientation of the vertical edges of one or more voussoirs are defined by the surface normal at each of those points (fig. 2.1 a). Consequently, a family of surface normals between those vertical edges defines a ruled surface. This can be achieved by considering all surface normals on a geodesic curve between two corresponding points on the surface (fig. $2.1 \mathrm{~b}$ ). Considering the fabrication constraints however, approximation through lofting between two vertical edge lines can be advantageous (fig. $2.1 \mathrm{c}$ ). First, the resulting doubly ruled surface can be traced by a wire in two directions, hence allowing more flexibility in the fabrication process. Second, the horizontal orientation of the generators allows for integrated interlocking notches, used for later reference during the assembly of the individual voussoirs (fig. $2.1 \mathrm{~d}$ ). Due to the relatively small surface curvature between two points, the deviation between both alternative surfaces (fig. $2.1 \mathrm{~b}$, c) can be neglected.

The previous approaches for generating the interface surfaces of voussoirs can typically not be applied in the same manner to the upper (extrados) and lower (intrados) surfaces of the voussoirs, due to their freeform, double-curved shape which can normally not be replaced by ruled surfaces, and hence, cannot be fabricated using a wire saw. A possible solution is to use an additional fabrication technology such as 5-axes milling or circular saw blade stone cutting. This will guarantee a smooth continuous extrados and intrados of the freeform stone vault (fig. 2.2 a). In contrast to the interface surfaces, the precision of the intrados and extrados is structurally of less importance, as long as the required vault thickness is guaranteed and thus can be approximated by ruled surfaces. This can be done by a global approximation of the intrados and extrados by strips of ruled surfaces [7]. This global approach would need to be highly coordinated with the tessellation in order to prevent generators of one ruled surface from intersecting with other parts of the voussoir geometry, especially for surfaces with negative curvature, as that would make the use of wire-cutting technology impossible.

An alternative, simple way to approximate the intrados and extrados locally is to segment the double-curved surfaces by two part surfaces. For the case of the hexagonal tessellation used as example in this paper, these are two quadrilateral surfaces (fig. $3.2 \mathrm{c}$ ). In the process shown in this paper, they will be automatically aligned to best fit the original geometry, regarding the distances between the midpoints to the corresponding closest points on the original intrados and extrados. If needed, this kinked geometry can be finished smoothly after installation by semimanual chiseling techniques.

\subsection{Machine Setup}

In order to provide the appropriate fabrication setup to process the geometry described above, different profiling machines have been examined in detail. They are equipped with a diamond wire to cut the stone within a variety of possible machine configurations. They range from single wire saws for dressing blocks and cutting slabs to computer-controlled systems for two- and three-dimensional 
profiling [11]. Those machines consist of standard elements, but customized configurations in terms of size and geometric flexibility are quite common. The most relevant machine configurations for stone processing based on wire-cutting technology can be categorized by the configuration and number of independent axes (fig. 2.2).

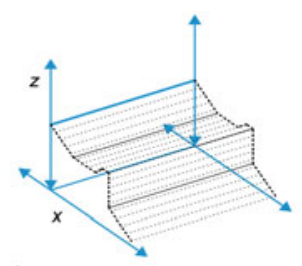

a

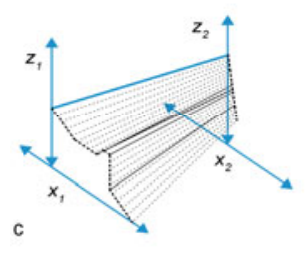

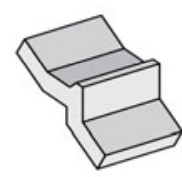

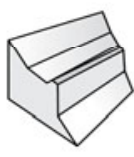

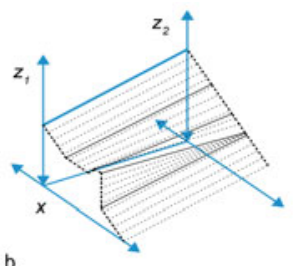
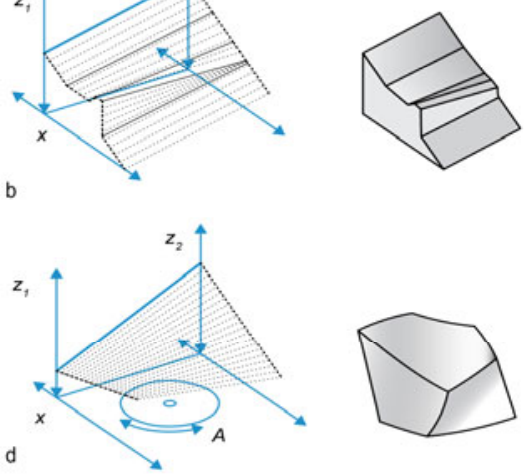

Fig. 2.2 (a) Profile cuts with a 2-axes machine (b) Conoid cuts with a 3-axes machine (c) Conical directed cuts with a 4-axes machine (d) Conical multidirectional cuts with a 4-axes machine

Simple stone profiling can be processed on a 2-axes machine setup (fig. 2.2 a), which enables planar cuts and is mostly used to cut rough slabs along the $X$ and $Z$ axes. More complex cuts are possible by using two independent vertical axes $\left(Z_{l}\right.$, $Z_{2}$ ) (fig. 2.2 b), e.g. to produce elements based on conoids where all rulings are parallel in planar projection. In contrast to the 2-axes setup, the wire length changes caused by asynchronous movement along the two vertical axes, raises the technical requirements for the wire guide and tensioning system.

For conical cuts a forth axis is needed (fig. $2.2 \mathrm{c}$ ). The combination of two individual horizontal axes $\left(X_{1}, X_{2}\right)$ and two individual vertical axes $\left(Z_{1}, Z_{2}\right)$ offers great geometrical flexibility, especially for linear elements. The increasing problem of the length differences for diagonal wire positions might be one reason why no wire saws based on this configuration could be found by the first author. In contrast, the same configuration is often found for CNC hot-wire cutters.

Volumetric parts with multidirectional orientation of ruled surfaces can be processed by the machine arrangement with only one horizontal axis $(X)$ and two individual axes in the vertical direction $\left(Z_{1}, Z_{2}\right)$, the forth axis $(A)$ in this case defines the rotation of a turntable (fig. $2.2 \mathrm{~d}$ ). Even more geometrical flexibility is possible using wire-cutting based on robotic arm fabrication $[12,13]$, but due to the limits of weight and picking mechanisms, the potential for this technique lies in hot-wire foam cutting, rather than in diamond-wire stone cutting and was therefore not being examined further. 


\section{Experimental Design and Fabrication Setup}

Considering the geometrical challenges of freeform stereotomy, the research shown in the previous chapter identifies the 4-axes machine configuration with one rotational axis (fig. $2.2 \mathrm{~d}$ ) has the most appropriate and efficient setup. The configuration allows the fabrication of complex volumes represented by patches of ruled surfaces. However, the relatively high investment needed to experiment with real stone cutting, led to the development of a customized $\mathrm{CNC}$ hot-wire cutter. It allows the exploration of automated foam cutting as a simulation of CNC stone processing. Hence, the ongoing research focuses on the geometrical constraints of those processes, rather than on the technical challenges of stone cutting related to the large weight of the material or the complexity of the abrasive cutting process.

Nevertheless, the possible size and shape of a voussoir in relation to the machine clearance, angle restrictions, and the stone volume, is very difficult to grasp. Therefore, a plugin for Rhinoceros 5.0 was developed to control conflicting parameters and visualize the result, respectively simulate the production process. This information was then evaluated and used to develop the machine setup and the prototypical voussoirs shown as a result of this research.

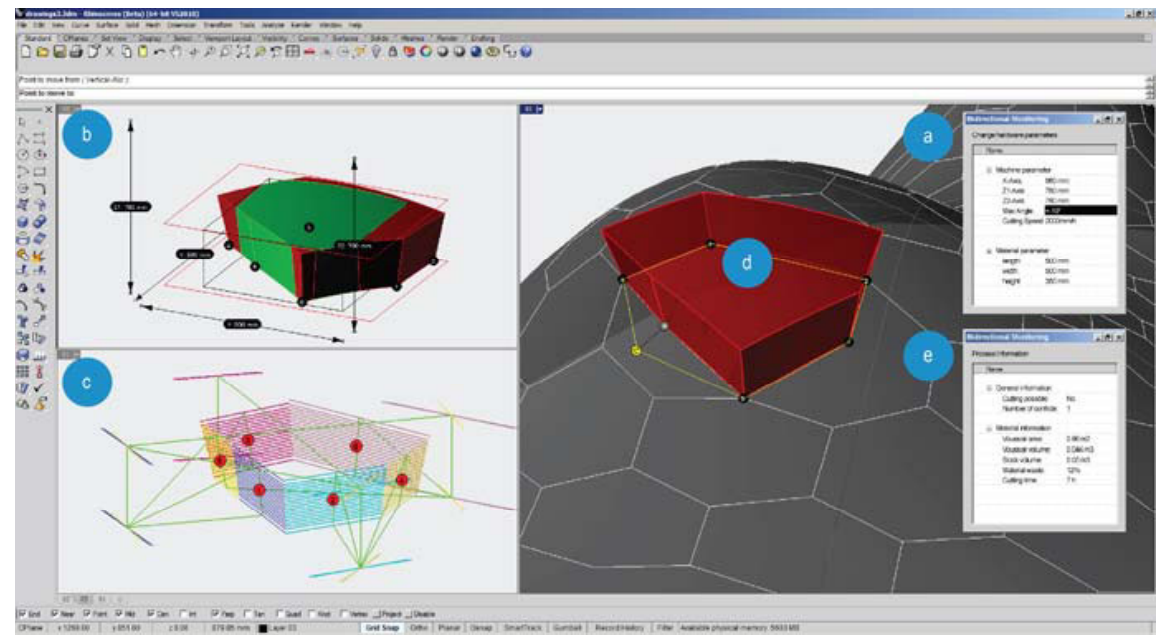

Fig. 3.1 While manipulating design, fabrication and material parameters, the production process is analyzed, visualized and simulated using a specially developed plugin for Rhinoceros 5.0

The screenshot shown in Figure 3.1 shows the CAD environment with the customized panel to control the parameters (fig. $3.1 \mathrm{a}$ ) and the visualization of the results (fig. $3.1 \mathrm{~b}, \mathrm{c}$ ). Machine parameters, such as individual axis length, maximum wire angle and the cutting speed, are defined numerically in a specific panel (fig. 3.1 a) but can also be manipulated by moving the endpoints of the axes in $3 \mathrm{~d}$ (fig. 3.1 b). The material dimensions can be changed in a similar way. 
Besides the virtual machine configuration and the processed voussoir (fig. 3.1 $\mathrm{b}, \mathrm{c}$ ), the tessellation and voussoir geometry on the thrust surface (fig. $3.1 \mathrm{~d}$ ) show the individual voussoir in the context of the overall vault geometry. This is used for real-time manipulation by changing the tessellation topology interactively on the thrust surface. This will affect the shape and dimension of the voussoir, which is automatically aligned and positioned within the virtual building chamber of the machine (fig. $3.1 \mathrm{~b}$ ). This real-time positioning is based on the minimum bounding box of the voussoir and the steepest generators of the corresponding ruled surfaces. The visualization of the aligned voussoir (fig. $3.1 \mathrm{~b}$ ), tool path information (fig. $3.1 \mathrm{c}$ ) and additional coloring, indicates possible fabrication conflicts for the chosen configuration. Moreover, the numerical output shows the number of conflicts, the amount of waste material and the approximated cutting time (fig. $3.1 \mathrm{e}$ ).

The developed program was used to verify and improve the design and fabrication setup described in this paper. In particular, the geometry of a previously developed freeform thin-tile vault [14] was used to test and verify the described methods, from the initial design to the final production, for the same geometry built from discrete stone blocks (fig. 3.2).
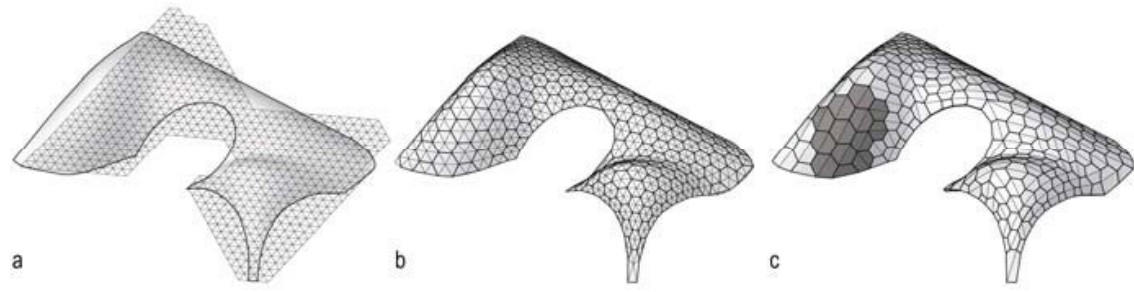

Fig. 3.2 (a) The NURBS surface and a planar network of triangular elements (b) The network gets evenly distributed on the surface using customized relaxation methods [9] (c) Depending on the curvature of the surface, the hexagonal and quadrilateral voussoirs are being generated

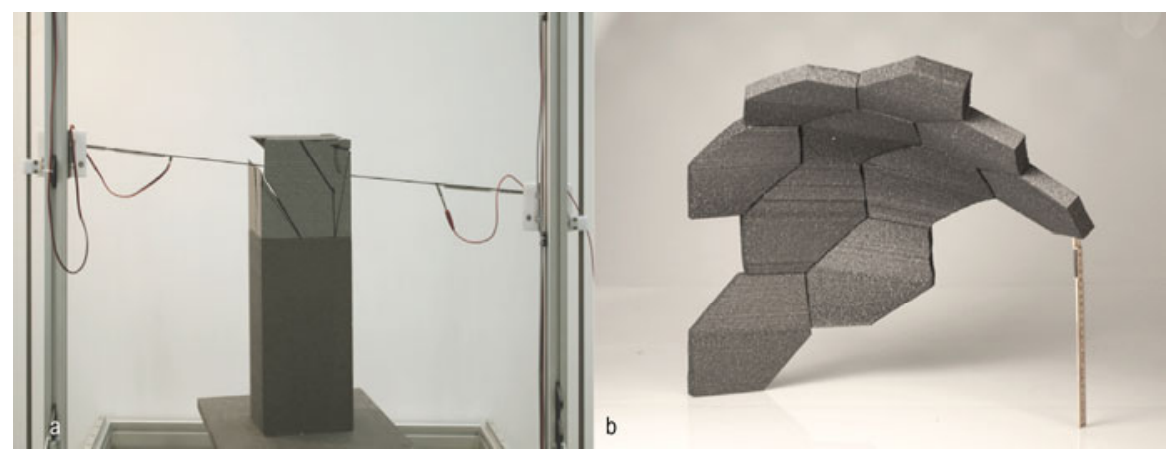

Fig. 3.3 (a) Shows the cutting process of a single voussoir. The Styrofoam block is mounted on the turntable, while the hot wire is travelling through the material. (b) This voussoir sample is one single piece of a patch of highly individual part geometries, also shown in fig. $3.2 \mathrm{c}$ 


\section{Conclusion}

This paper has described a powerful approach and technique to materialize stone vaults, considering today's high demands on feasibility and effectiveness. A strategy for generating individual and geometrically complex voussoirs has been developed, taking into account architectural, structural and fabrication requirements. An appropriate fabrication setup in combination with the design method was developed and further analyzed and improved using a custom-made plugin for the visualization of the complex interdependencies of design, fabrication and material parameters. Thanks to this program, it was possible to demonstrate that different freeform vault designs can be generated and produced based on ruled surface geometry. Hence, allowing the use of hot-wire cutting, which persuasively illustrates the possibilities for real stone cutting. Moreover, the real-time feedback about relevant fabrication data helped to control the feasibility of the process prior production and finally facilitated the technical realization.

Future research will focus on an automated feedback system for the voussoir generation, which not only visualizes the limitations of the fabrication, but automatically finds the best possible configuration considering specific criteria. This process could be done iteratively by using genetic algorithms or nonlinear optimization methods. Therefore, the speed and real-time performance of the program needs to be improved. Concerning the realization of real stone vaults, possible industry partners have already been contacted to work on the technical implementation of the stone-cutting process.

\section{References}

[1] Glymph, J., et al.: A parametric strategy for free-form glass structures using quadrilateral planar facets. Automation in Construction 13(2), 187-202 (2004)

[2] Evans, R.: The Projective Cast. MIT Press, Cambridge (1995)

[3] Schodek, D., Bechthold, M., et al.: Digital Design and Manufacturing: CAD/CAM Ap-plications in Architecture and Design. Wiley, Hoboken (2005)

[4] Lordick, D.: Intuitive Design and Meshing of Non-Developable Ruled Surfaces. In: Proceedings of the Design Modelling Symposium 2009, October 5-7, pp. 248-261. University of the Arts, Berlin (2009)

[5] Burry, M., et al.: Gaudí unseen: completing the Sagrada Família. Jovis, Berlin (2007)

[6] Asche, J.: Tiefschleifen von Granit. University of Hannover, Dissertation (2000)

[7] Flöry, S., Pottmann, H.: Ruled Surfaces for Rationalization and Design in Architecture. In: Proceedings of ACADIA 2010, pp. 103-109. The Cooper Union, New York (2010)

[8] Block, P.: Thrust Network Analysis: Exploring Three-dimensional Equilibrium. Dissertation, Massachusetts Institute of Technology, Cambridge (2009)

[9] Lachauer, L., Rippmann, M., Block, P.: Form Finding to Fabrication: A digital design process for masonry vaults. In: Proceedings of the International Association for Shell and Spatial Structures (IASS) Symposium 2010, Shanghai (2010) 
[10] Rippmann, M., Block, P.: Digital Stereotomy: Voussoir geometry for freeform masonry-like vaults informed by structural and fabrication constraints. In: Proceedings of the International Association for Shell and Spatial Structures (IASS) Symposium 2011, London (2011)

[11] Pellegrini: Robot Wire Stone Profiling. Catalogue Profling Machines (2011), http://www.pellegrini.net/cataloghi/PELLEGRINI_RobotWire.pdf (accessed June 10, 2011)

[12] Gramazio, F., Kohler, M.: Designers Saturday. Design exhibition, Langentahl, Digital Fabrication in Architecture, ETH, Zurich (2010), http://www.dfab.arch.ethz.ch/web/d/forschung/191.html (accessed June 10, 2011)

[13] Meier, M.: Robotic Fabrication of Parametric Chairs. Taubman College of Architecture. University of Michigan, Ann Arbor (2011),

http://mkmra2.blogspot.com/2011/04/robotic-fabrication-of-parametric.html

[14] Davis, L., Rippmann, M., Pawlowfsky, T., Block, P.: Efficient and Expressive Thintile Vaulting using Cardboard Formwork. In: Proceedings of the International Association for Shell and Spatial Structures (IASS) Symposium 2011, London (2011) 